\title{
Comparative Efficacy of the Combination of Topical Betamethasone Dipropionate and Calcipotriene with Betamethasone Dipropionate and Calcipotriene Alone in the Treatment of Localized Vitiligo.
}

\author{
Alam MN ${ }^{1}$, Wahab MA², Husain $\mathrm{MA}^{3}$, Khondker L ${ }^{4}, \mathrm{Ahmad} \mathrm{GKA}^{5}$, Chakraborty ${ }^{6}$
}

\begin{abstract}
Vitiligo is an acquired skin disorder characterized by welldefined white patches that are often symmetrically distributed.

The study was conducted to compare the efficacy of the combination of topical betamethasone dipropionate and calcipotriene with betamethasone dipropionate and calcipotriene alone in the treatment of localized vitiligo.

A clinical trial was carried out with the patients of vitiligo from January 2012 to August 2012. In group A, 20 patients were applied betamethasone dipropionate cream $0.05 \%$ and topical calcipotriene ointment (0.005\%), in group $B$, 20 patients were applied betamethasone dipropionate cream $0.05 \%$ only; In group C, 20 patients were applied calcipotriene ointment $0.005 \%$ alone.
\end{abstract}

From base line percentage reduction of total lesion in $1^{\text {st }}$ follow up in group A, group B and group $C$ were 20\%, 15\% and $10 \%$ respectively. At $3^{\text {rd }}$ follow up in group $A$, group $B$ and group $C$ it was $50 \%, 37 \%$ and $30 \%$ and at $5^{\text {th }}$ follow up it was $80 \%$, 75\% and $65 \%$ respectively. ANOVA test was done and found significant difference of reduction of total lesion of vitiligo among the groups $(p<0.05)$. At the completion of the study, each patient was separately graded the treated sides

1. Corresponding Author : Dr. Mohd. Nurul Alam MBBS, FCPS, DDV, MCPS.

Assistant Professor, Department of Dermatology \& Venereology Ibn Sina Medical College \& Hospital, Kallyanpur, Dhaka

2. Professor Lt. Col (Retd.) Dr. Md. Abdul Wahab MBBS, FCPS, DDV, MCPS, FRCP.

Professor, Department of Dermatology \& Venereology Bangabandhu Sheikh Mujib Medical University, Dhaka

3. Dr. Major (Retd.) Md Anwar Husain MBBS, DDV, MCPS.

Associate Professor, Department of Dermatology \& Venereology Ibn Sina Medical College \& Hospital, Kallyanpur, Dhaka

4. Dr. Lubna Khondker MBBS, FCPS, DDV, MCPS, MPH. Assistant Professor, Department of Dermatology \& Venereology Bangabandhu Sheikh Mujib Medical University, Dhaka

5. Dr. Gulam Kazem Ali Ahmad MBBS, FCPS, DDV Specialist in Dermatology and Venereology Bangabandhu Sheikh Mujib Medical University, Dhaka

6. Dr. Anjana Chakraborty MBBS, DDV Specialist in Dermatology and Venereology Bangabandhu Sheikh Mujib Medical University, Dhaka on a 6-point ordinal scale based on a global estimate of the change in vitiligo and found that, the very much improvement were $70 \%$, 55\% and 45\%, much improvement were 20\%, $30 \%$ and $35 \%$ and improvement were $10 \%, 15 \%$ and $20 \%$ in group $A$, group B and group $C$ respectively. ANOVA test was found significant difference of success rate of patients of localized vitiligo among the groups ( $p$ value 0.005).

Both the drugs, calcipotriene and betamethasone dipropionate when used individually, were found to be equally effective in the treatment of vitiligo, but the combination of the two was found to be superior in efficacy.

Key words : Betamethasone dipropionate, calcipotriene, vitiligo.

\section{Introduction}

Vitiligo vulgaris is a pigmentary disorder with no universally efficacious therapeutic options. It is an acquired skin disorder that result from damage to and destruction of melanocytes. ${ }^{1}$ The cause is unknown but may involve genetic factors, autoimmunity, toxic metabolites and/or a higher vulnerability of melanocytes. ${ }^{2-4}$ It is affecting $0.5-2 \%$ of the population worldwide. ${ }^{5,6}$ Vitiligo can be cosmetically disfiguring and it is a stigmatizing condition, leading to serious psychologic problems in daily life. ${ }^{7,8}$ Two of the major theories of the pathogenesis of vitiligo are the autoimmune theory and the autocytotoxicity theory. ${ }^{9}$ The autoimmune theory speculates that patients with vitiligo form autoantibodies against melanocytes. The existence of antimelanocyte surface antigen antibodies has been demonstrated, and the severity of vitiligo has proven to be related to the amount of antibodies present. ${ }^{10,11}$ Patients have numerous treatment options available, but none is universally effective. Even among patients who respond to treatment there is a high potential for relapse. Treatment of vitiligo is a challenge. ${ }^{12}$ The most widely prescribed therapies are PUVA and topical corticosteroids. PUVA is not recommended for children because of concern over its long term side effects, and prolonged use of topical steroids has the potential to cause cutaneous atrophy, including telangiectasis and perioral dermatitis. ${ }^{13}$

Calcipotriene is a synthetic analog of vitamin $\mathrm{D}_{3}$ (calcitriol: 1 , $\left.25(\mathrm{OH})_{2} \mathrm{D}_{3}\right)$ that has been shown to have immunomodulating and immunosuppressive actions. ${ }^{14}$ Receptors for $1,25(\mathrm{OH})_{2} \mathrm{D}_{3}$ the active form of vitamin $\mathrm{D}$, have been demonstrated on keratinocytes, melanocytes and fibroblasts, and on immunologically active cells. Melanocytes and keratinocytes within vitiligenous lesions have shown defective calcium uptake. Based on this observation, recently investigators demonstrated that calcipotriene can be effective for vitiligo, both as monotherapy and in combination in Bangladeshi population. ${ }^{15,16}$ 


\section{Materials and Methods}

It was a clinical trial, from January 2012 to August 2012. Patients of vitiligo attending outpatient department of Dermatology and Venereology, Bangabandhu Sheikh Mujib Medical University (BSMMU), Dhaka were the study population and non-probability sampling method was followed in this study. Inclusion criterias were all localized vitiligo patients, age more than 5 years of both sexes. The exclusion criterias were generalized vitiligo patients, previous skin malignancy, treatment for vitiligo with corticosteroid agents, vitamin D analogues, or tacrolimus within the last 3 months, pregnancy and lactation, renal or hepatic disease, lupus erythematosus, severely ill patients and patients or attendants unwilling to take part in the study.

\section{Procedures of collecting data}

A total of 60 patients suffering from localized vitiligo were primarily selected from outdoor department of Dermatology \& Venereology department, BSMMU. Complete history, general physical, dermatological and Wood's light examinations were done for all enrolled patients. For women of reproductive age, reproductive history were carefully mentioned. History and physical findings were recorded in a structured questionnaire. Finally those patients, who were matched the inclusion and exclusion criteria according to history, physical examination and freely gave their informed consent, were selected for study. Clinical assessment at baseline and follow up visit, monthly for 5 months were taken and photographs of all lesions at baseline and after 5 months were taken for subsequent assessment and further comparison. The patients were randomized into three treatment groups. In group A, 20 patients were applied betamethasone dipropionate cream $0.05 \%$ in the morning and topical calcipotriene ointment $(0.005 \%)$ in the evening, group B, 20 patients with betamethasone dipropionate cream $0.05 \%$ twice daily; In group C, 20 patients were applied calcipotriene ointment $0.005 \%$ similarly; and each individual lesion were treated daily for five months. Generally the efficacy of repigmentation therapy \& safety were recorded every four weekly for five months. Patient data were recorded on pre-designed case record form.

Efficacy was assessed by score of VASI (Vitiligo Area Scoring Index). The first involved structured monthly estimation of body surface area vitiligo involvement using the VASI. The body was divided into 5 separate and mutually exclusive regions: hands, upper extremities (excluding hands), trunk, lower extremities (excluding the feet), and feet. The axilliary and inguinal regions were included with the upper and lower extremities, respectively, while the buttocks were included with the lower extremities. The face and neck areas were assessed and treated for vitiligo if requested by the patient, but these areas were not included in the overall evaluation. One hand unit, which encompasses the palm plus the volar surface of all the digits, is approximately $1 \%$ of the total body surface area and was used as a guide to estimate the baseline percentage of vitiligo involvement of each body region. To eliminate variations in hand size, we defined a hand unit to be the volar hand, including fingers.

At each follow-up assessment, any macular repigmentation was noted, and the extent of residual depigmentation within each affected patch that had been present at baseline was estimated to the nearest of 1 of the following percentages: 0 , $10 \%, 25 \%, 50 \%, 75 \%, 90 \%$, or $100 \%$. Any new depigmented patches that developed during the study were also estimated using the hand unit method and were included in the VASI calculation. Standardized assessments for estimating the degree of pigmentation to derive the Vitiligo Area Scoring Index. At $100 \%$ depigmentation, no pigment is present; at $90 \%$, specks of pigment are present; at $75 \%$, the depigmented area exceeds the pigmented area; at $50 \%$, the depigmented and pigmented areas are equal; at $25 \%$, the pigmented area exceeds the depigmented area; and at $10 \%$, only specks of depigmentation are present. For the second measurement method, total body photographs were taken at baseline and at each monthly follow-up visit as an aid to the global clinical scoring. These $35-\mathrm{mm}$ slides were used by investigators for global assessments, which were done halfway through and at the end of the study.

For each body region, the VASI was determined by the product of the area of vitiligo in hand units (which were set at $1 \%$ per unit) and the extent of depigmentation within each hand unit-measured patch (possible values of $0,10 \%, 25 \%$, $50 \%, 75 \%, 90 \%$, or $100 \%$ ). The total body VASI was then calculated using the following formula by considering the contributions of all body regions (possible range, $0-100$ ):

VASI $=\sum_{\substack{\text { All Body } \\ \text { Sites }}}[$ Hand Unis $] X$ [Residual Depigmentation $]$

To compare the VASI system with ordinal scales analogous to those used in previous studies, we performed global assessments. At the completion of the study, each patient separately graded the treated sides on a 6-point ordinal scale based on a global estimate of the change in vitiligo as follows: complete improvement (100\%), very much improved (76\%$99 \%$ ), much improved (51\%-75\%), improved (26\%-50\%), minimal change (1\%-25\%), no change. Data analysis was performed by Statistical Package for Social Science (SPSS), and level of significance was measured by using appropriate statistical test and level of significance ( $p$ value) was set at 0.05 and confidence interval at $95 \%$.

\section{Results}

Mean age of group A, group B and group C patients were $21.50 \pm 3.32,21.55 \pm 4.12$ and $22.25 \pm 4.67$ respectively. There was no significant difference of age between the groups. 
Table I: Distribution of age of the patients by groups.

\begin{tabular}{ccccc}
\hline $\begin{array}{c}\text { Age (in } \\
\text { years) }\end{array}$ & $\begin{array}{c}\text { GroupA } \\
(\mathbf{n = 2 0 )}\end{array}$ & $\begin{array}{c}\text { GroupB } \\
(\mathbf{n = 2 0})\end{array}$ & $\begin{array}{c}\text { Group C } \\
(\mathbf{n = 2 0 )}\end{array}$ & $\begin{array}{c}\text { *p } \\
\text { value }\end{array}$ \\
\hline $13-22$ & $7(35 \%)$ & $8(40 \%)$ & $7(35 \%)$ & 0.810 \\
$23-32$ & $9(45 \%)$ & $6(30 \%)$ & $6(30 \%)$ & \\
$>32$ & $4(20 \%)$ & $6(30 \%)$ & $7(35 \%)$ & \\
Mean \pm SD & $21.50 \pm 3.32$ & $21.55 \pm 4.12$ & $22.25 \pm 4.67$ & \\
\hline
\end{tabular}

* ANOVA test done to measure significance

In distribution of the patients by main site of lesion in different groups, extremities involvement were $60 \%, 70 \%$ and $45 \%$, face involvement were $30 \%, 20 \%$ and $35 \%$, and trunk involvement were $10 \%, 10 \%$ and $20 \%$ in group A, group B and group $\mathrm{C}$ respectively. ANOVA test was done to measure significance and found no significant difference of main site of lesion among the groups ( $\mathrm{p}>0.05)$.
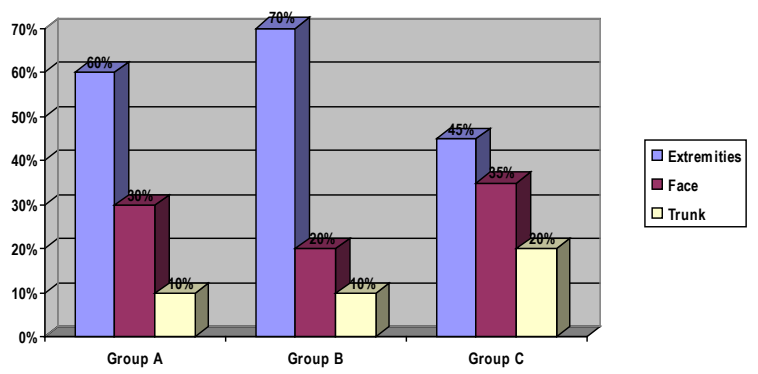

Figure 1: Distribution of the patients by main site of lesion in different groups.

In distribution of the patients by duration of lesion in different groups, less than 1 year duration were $45 \%, 70 \%$ and $35 \%$ and more than 1 year duration were $55 \%, 30 \%$ and $65 \%$ in group A, group B and group C respectively. ANOVA test was done to measure significance and found no significant difference of main site of lesion among the groups ( $p>0.05)$.

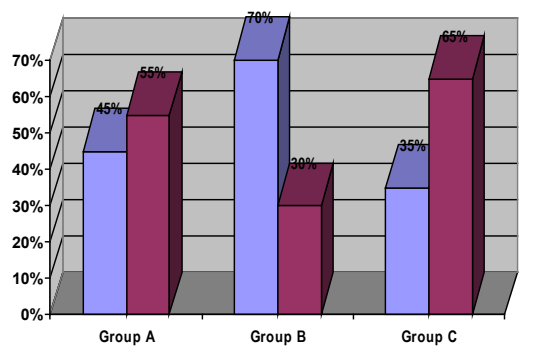

$\square$ Less than 1 year $\square$ More than 1 year

Figure 2: Distribution of the patients by duration of lesion in different groups.

At base line the score of vitiligo in group A, group B and group $\mathrm{C}$ were 26,25 and 23 respectively. At $1^{\text {st }}$ follow up it was 20, 22 and 19, at $3^{\text {rd }}$ follow up it was 10,15 and 12 , and at 5th follow up it was 3,8 and 6 in group A, group B and group $\mathrm{C}$ respectively. ANOVA test was done to measure significance and found significant difference of score of vitiligo among the groups $(\mathrm{p}<0.05)$.

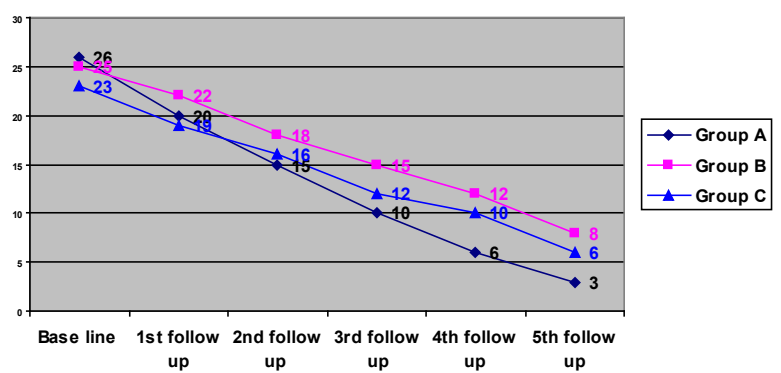

Figure 3: Total score of progressive follow ups in different groups

From base line percentage reduction of total lesion in $1^{\text {st }}$ follow up were $20 \%, 15 \%$ and $10 \%$, at $3^{\text {rd }}$ follow up it was $50 \%, 37 \%$ and $30 \%$, and at $5^{\text {th }}$ follow up it was $80 \%, 75 \%$ and $65 \%$ in group A, group B and group C, respectively. ANOVA test was done to measure significance and found significant difference of reduction of total lesion of vitiligo among the groups $(\mathrm{p}<$ $0.05)$.

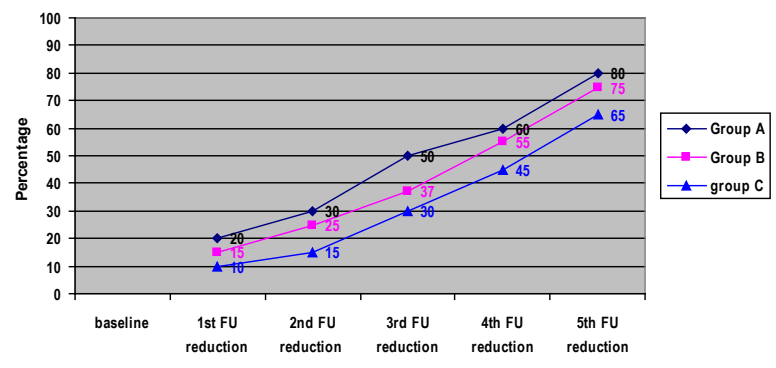

Figure 4: line chart of percentage reduction of total lesion

At the completion of the study, each patient was separately graded the treated sides on a 6-point ordinal scale based on a global estimate of the change in vitiligo and found that, the very much improvement were $70 \%, 55 \%$ and $45 \%$, much improvement were $20 \%, 30 \%$ and $35 \%$ and improvement were $10 \%, 15 \%$ and $20 \%$ in group A, group B and group C respectively. ANOVA test was found significant difference of success rate of patients of localized vitiligo among the groups (p value 0.005 ).

Table II: Success rate of patients of localized vitiligo

\begin{tabular}{lcccc}
\hline \multirow{1}{*}{ Success rate } & \multicolumn{4}{c}{ Groups } \\
\cline { 2 - 4 } & $\begin{array}{c}\text { Group A } \\
(\mathbf{n = 2 0})\end{array}$ & $\begin{array}{c}\text { Group B } \\
(\mathbf{n = 2 0})\end{array}$ & $\begin{array}{c}\text { GroupC } \\
(\mathbf{n = 2 0})\end{array}$ & * v value \\
\hline Complete-improvement & 0 & 0 & 0 \\
Very much improved & $14(70 \%)$ & $11(55 \%)$ & $9(45 \%)$ & \\
Much improved & $4(20 \%)$ & $6(30 \%)$ & $7(35 \%)$ & 0.005 \\
Improvement & $2(10 \%)$ & $3(15 \%)$ & $4(20 \%)$ & \\
minimal change & 0 & 0 & 0 & \\
no change & 0 & 0 & 0 \\
\hline
\end{tabular}

\section{Discussion}

At $12^{\text {th }}$ week of therapy, the very much improvement were $70 \%, 55 \%$ and $45 \%$, much improvement were $20 \%, 30 \%$ 
and $35 \%$, improvement were $10 \%, 15 \%$ and $20 \%$ in group $\mathrm{A}$, group $\mathrm{B}$ and group $\mathrm{C}$ respectively ( $\mathrm{p}$ value 0.005 ). These findings were consistent with other studies like Kumaran et al and Parsad et al. ${ }^{15,16}$ A trial was conducted by Kumaran et al to evaluate the effect of topical calcipotriol ointment $(0.005 \%)$ and betamethasone dipropionate $(0.05 \%)$ cream, given alone or in combination, in treatment of localized vitiligo. Group I patients were treated with betamethasone dipropionate $(0.05 \%)$ cream twice daily. Group II patients were treated with calcipotriol ointment $(0.005 \%)$ twice daily, and group III with betamethasone dipropionate $(0.05 \%)$ in the morning and calcipotriol $(0.005 \%)$ in the evening. Marked (50\% to $75 \%$ ) repigmentation was observed in 2 $(13.3 \%), 1(6.7 \%)$ and $4(26.7 \%)$ patients in groups I, II and III, respectively and moderate $(25-50 \%)$ repigmentation was observed in 7 (46.7\%), 5 (33.3\%) and 7 (46.7\%) patients in groups I, II and III, respectively. They concluded that combined therapy appeared to give a significantly faster onset of repigmentation along with better stability of the achieved pigmentation. ${ }^{15}$ Parsad et al enrolled twenty-one patients age 5 to 17 years with vitiligo in this study and the children were advised to apply calcipotriol $50 \mathrm{microg} / \mathrm{g}$ in the evening and expose themselves to sunlight the next day for 10 to 15 minutes. Initial repigmentation occurred in the majority of children after 6 to 12 weeks of treatment. Marked to complete repigmentation was seen in 10 of 18 patients and four patients showed moderate improvement. ${ }^{16}$

Our study observed that both the drugs, calcipotriol and betamethasone dipropionate when used individually as monotherapy, were found to be equally effective in the treatment of vitiligo, but the combination of the two was found to be superior in efficacy. These findings of our study were not consistent with study findings of Chiavérini et al. ${ }^{17}$ Chiavérini et al carried out a study to evaluate the efficiency of topical calcipotriol monotherapy in vitiligo and concluded that topical calcipotriol in monotherapy is not an effective treatment of vitiligo. Our study findings were also consistent with findings of Pasricha et al. ${ }^{18}$ In my study, both the drugs, calcipotriol and betamethasone dipropionate when used individually, were found to be effective in the treatment of vitiligo. Pasricha et al tried a new approach using mini-pulse therapy with betamethasone. Forty patients having extensive and/or fast-spreading vitiligo were given $5 \mathrm{mg}$ betamethasone/dexamethasone as a single oral dose after breakfast on 2 consecutive days per week. Within 1-3 months, progression of the disease was arrested in $89 \%$ of the 36 patients having active disease, while 2 patients needed an increase in the dose to $7.5 \mathrm{mg}$ per day to achieve complete arrest of lesions. Within 2-4 months, $80 \%$ of the patients started having spontaneous repigmentation of the existing lesions which progressed with continued treatment. The extent of repigmentation varied in different patients and even in different lesions in the same patient. It was less than $10 \%$ in $14(35 \%)$ patients and almost complete $(>90 \%)$ in three patients. Oral mini-pulse therapy with betamethasone seems to be an effective treatment modality to arrest the progression of vitiligo. It also induces spontaneous repigmentation. ${ }^{18}$

Both the drugs, calcipotriene and betamethasone dipropionate when used individually, were found to be equally effective in the treatment of vitiligo, but the combination of the two was found to be superior in efficacy.

\section{References}

1. Njoo MD, Westerhof W. Vitiligo. Pathogenesis and treatment. Am J Clin Dermatol .2001;2:167-8.

2. Westerhof W. Vitiligo management update. Skin Therapy Lett. 2000; 5:1-2,5

3. Bleehen SS. Disorders of skin color. In: Champion RH, Burton JL, Burn DA, Breathnach SM eds. Rook/ Wikinson/ Ebling textbook of Dermatology. London Blackwell Science. 1998;1753-1815.

4. Kovacs SO. Vitiligo. J Am Acad Dermatol. 1998;38:64766.

5. Halder RM, Taliaferro SJ. Vitiligo. Wolff K, Goldsmith LA, Katz SI, Gilchrest BA, Paller AS and Leffell DJ. Fitzpatrick's Dermatology in General Medicine. New York: The McGraw-Hill Companies. 2008;616-8.

6. Aamir Habib, Zafar Iqbal Sheikh, Qamarud Din Khan, Simeen Ber Rahman. Efficacy and safety of oral dexamethasone pulse treatment for vitiligo. Pakistan Armed Forces Medical Journal. 2006;2:1-6

7. Lebwohl M. Topical application of calcipriene and corticosteroid: combination regimens. $J$ Am Acad Dermatol. 1997;37:55-58.

8. Travis LB, Silverberg NB. Calcipotrien and corticosteroid combination therapy for vitiligo. Pediatric Dermatology. 2004;21:495-498.

9. Kim SM, Lee HS, Hann SK. The efficacy of low-dose oral corticosteroids in the treatment of vitiligo patients. Int J Dermatol. 1999;38:546-50.

10. Pasricha JS, Khaitan BK. Oral mini-pulse therapy with betamethasone in vitiligo patients having extensive or fast spreading disease. Int J Dermatol. 1993;32:753-7.

11. Radakovic-Fijan S, Furnsinn-Friedl AM, Honigsmann $\mathrm{H}$, Tanew A Oral dexamethasone pulse treatment for vitiligo. J Am Acad Dermatol. 2001;44:814-7.

12. Seister S, Ugruel S, Tilgen W, Reinhold U. Use of high dose methylprednisolone pulse therapy in patients with progressive and stable vitiligo. Int J Dermatol. 2000;39:624-7.

13. Majid I. Vitiligo Management: An Update. BJMP. 2010;3:332

14. Lisa BT, Nanette BS. Calcipotriene and Corticosteroid 
combination therapy for vitiligo. Pediatric Dermatology. 2004;21:495-498.

15. Kumaran MS, Kaur I, Kumar B. Effect of topical calcipotriol, betamethasone dipropionate and their combination in the treatment of localized vitiligo. Journal of the European Academy of Dermatology and Venereology. 2006;20:269-273.

16. Parsad D, Saini RDNB, Nagpal R. Calcipotriol in vitiligo: a preliminary study. Pediatr Dermatol. 1999;16:317-20.
17. Chiavérini $\mathrm{C}$, Passeron $\mathrm{T}$, Ortonne JP. Treatment of vitiligo by topical calcipotriol. Journal of the European Academy of Dermatology and Venereology. 2002;16:137-138.

18. Pasricha JS, Khaitan BK. Oral mini-pulse therapy with betamethasone in vitiligo patients having extensive or fast spreading disease. Int J Dermatol. 1993;32:753-7. 